\title{
MANAGEMENT OF HYPERLIPIDEMIA IN VERY HIGH AND EXTREME RISK PATIENTS IN CROATIA: AN OBSERVATIONAL STUDY OF TREATMENT PATTERNS AND LIPID CONTROL
}

\author{
Hrvoje Pintarić1,2, Marijana Knezović Florijan², Ian Bridges ${ }^{3}$, Robert Steiner ${ }^{4}$, \\ Luka Zaputović ${ }^{5}$ and Davor Miličićc
}

\author{
${ }^{1}$ University of Zagreb, School of Dental Medicine, Zagreb, Croatia; \\ ${ }^{2}$ Sestre milosrdnice University Hospital Centre, Department of Internal Medicine, Zagreb, Croatia; \\ ${ }^{3}$ Amgen Ltd., Cambridge, United Kingdom; \\ ${ }^{4}$ Osijek University Hospital Centre, Department of Cardiology, Osijek, Croatia; \\ ${ }^{5}$ Rijeka University Hospital Centre, Rijeka, Croatia; \\ ${ }^{6}$ University of Zagreb, School of Medicine, Department of Cardiovascular Diseases, \\ Zagreb University Hospital Centre, Zagreb, Croatia
}

\begin{abstract}
SUMMARY - Our observational study evaluated current management of elevated low-density lipoprotein cholesterol (LDL-C) in adult secondary prevention patients (all very high risk (VHR) by European guidelines) attending specialist clinics across Croatia. Data were collected retrospectively from patient records for the preceding 12 months. The subset judged to be at extreme risk (ER; American Association of Clinical Endocrinologists (AACE) criteria; $n=48$ ) were compared with the remaining patients (VHR group; $\mathrm{n}=41$ ). All patients were receiving statins $(75.6 \% \mathrm{VHR} / 81.3 \% \mathrm{ER}$ at high-intensity), with only a minority receiving concomitant lipid-lowering treatment $(7.3 \%$ VHR $/ 16.7 \%$ ER). Median (Q1, Q3) LDL-C levels at the last visit were $1.9(1.6,2.4) \mathrm{mmol} / \mathrm{L}$ for VHR and $2.1(1.5,3.1) \mathrm{mmol} / \mathrm{L}$ for ER, with only $41.5 \%$ (95\% CI 26.3-57.9) of VHR patients and $27.1 \%$ (15.3-41.9) of ER patients attaining their LDL-C targets $(<1.8 \mathrm{mmol} / \mathrm{L}$ and $<1.42 \mathrm{mmol} / \mathrm{L}$, respectively). Thus, we found that a substantial proportion of VHR and ER secondary prevention patients being treated across Croatia had LDL-C levels exceeding the targets recommended in the European and newer AACE guidelines, but not all were receiving high-intensity statins. Identification of ER patients and their lipid patterns may help optimize usage of high-intensity statin treatment, alone or along with newer treatments, for better control of elevated LDL-C.
\end{abstract}

Key words: Hyperlipidemia; Secondary prevention; High-risk patients; Very high-risk patients; Extreme risk patients

\section{Introduction}

Dyslipidemia is a primary major risk factor for atherosclerotic cardiovascular disease (ASCVD), the

Correspondence to: Hrvoje Pintaric, $M D, P h D$, School of Dental Medicine, University of Zagreb, Gundulićeva 5, HR-10000 Zagreb, Croatia

E-mail: pintaric.hrvoje@gmail.com

Received October 1, 2019, accepted August 28, 2020 leading cause of death and disability in the developed world ${ }^{1}$. Lipid-lowering therapy (LLT) aimed at reducing elevated low-density lipoprotein cholesterol (LDL-C), the dominant form of atherogenic cholesterol, substantially reduces the risk of cardiovascular $(\mathrm{CV})$ events in the primary and secondary prevention settings ${ }^{2-4}$. Statin drugs have provided the backbone of LLT for several decades. 
Given that the degree of risk reduction is proportional to the magnitude of LDL-C reduction ${ }^{5}$, the $\mathrm{Eu}-$ ropean Society of Cardiology/European Atherosclerosis Society (ESC/EAS) ${ }^{6,7}$ have set specific LDL-C targets for patients with hyperlipidemia, according to their CV risk. In 2011, an LDL-C target of $<2.5$ $\mathrm{mmol} / \mathrm{L}$ was recommended for high risk (HR) and $<1.8 \mathrm{mmol} / \mathrm{L}$ for very high risk (VHR) patients ${ }^{6}$, with the HR goal being subsequently revised to $<2.6$ $\mathrm{mmol} / \mathrm{L}$ in $2016^{7}$. More recently, a new risk category, extreme risk (ER), has been defined in guidelines from the American Association of Clinical Endocrinologists (AACE) and American College of Endocrinology (ACE) as "progressive atherosclerotic cardiovascular disease (ASCVD) after achieving LDL-C $<1.8$ $\mathrm{mmol} / \mathrm{L}$; established clinical $\mathrm{CV}$ disease in patients with diabetes mellitus, chronic kidney disease (CKD) stage $3 / 4$, or heterozygous familial hypercholesterolemia $(\mathrm{FH})$; history of premature ASCVD (male aged $<55$ years and female aged $<65$ years)" ${ }^{8}$. Individuals falling into this category have a significantly increased risk of a major or fatal ASCVD event over the next 4-5 years. An LDL-C target of $<1.42 \mathrm{mmol} / \mathrm{L}$ has been advocated for such patients ${ }^{8}$, on the basis of findings from large studies such as IMPROVE-IT (examining outcomes in subjects with acute coronary syndrome: Vytorin vs. simvastatin) ${ }^{9}$ and FOURIER (further cardiovascular outcomes research with PCSK9 inhibition in subjects with elevated risk $)^{10}$, showing that lowering LDL-C to the levels below previous targets provided additional outcome benefits.

Data from observational studies and surveys in Western Europe and the US indicate that LDL-C targets are not being met in a substantial proportion of patients treated for dyslipidemia ${ }^{11,12}$. A retrospective/ prospective observational study was conducted to explore the management of dyslipidemia across several Centra1/Eastern European countries and Israel, with a focus on treatment patterns and LDL-C target achievements ${ }^{13}$. Here we present results for the Croatian subpopulation.

\section{Patients and Methods}

Briefly, this regional study was a retrospective/prospective non-interventional, observational study that enrolled adult patients diagnosed with hyperlipidemia who were receiving LLT and attending a specialist (cardiologist, diabetologist) and/or internist for rou- tine visit ${ }^{13}$. Patients receiving LLT in the course of an interventional clinical trial were excluded. Data were collected from individual patient medical records for 12 months prior to enrolment (retrospective phase; all countries) and up to 6 months following enrolment (prospective phase; in Romania, Poland and the Czech Republic only). Patients were required to have at least 2 LDL-C values and valid LLT documentation (type of medication and dose) for the retrospective data collection period and provided informed consent where required by local regulations.

Our Croatian centers enrolled only VHR patients, classified according to the European guidelines ${ }^{6}$. Individuals who fulfilled the criteria for the newly defined ER category proposed by $\mathrm{AACE} / \mathrm{ACE}^{8}$ were identified and their data compared with the remaining patients (designated as the VHR group).

\section{Objectives}

The primary study objective was to estimate the proportion of patients achieving recommended LDL-C target levels of $<1.8 \mathrm{mmol} / \mathrm{L}$ for $\mathrm{VHR}^{6}$ and $<1.42 \mathrm{mmol} / \mathrm{L}$ for $\mathrm{ER}^{8}$. Secondary objectives included LDL-C levels over time; use of statins and other LLT (type, dose, frequency) over time; $\mathrm{CV}$ events; clinical characteristics (demographics, medical history including $\mathrm{CV}$ events, symptoms of statin intolerance, hospitalizations); and parameters of clinical management of patients. No formal hypothesis was tested.

\section{Study sample size}

It was planned to enroll a total of 80-150 subjects per country in the regional study, based on the projected $50 \%$ of subjects achieving the primary outcome measure (achievement of ESC/EAS LDL-C targets). Thus, a sample size of 80 patients would enable the percentage to be estimated with $95 \%$ confidence interval $(\mathrm{CI})$ with a half-width of $11.0 \%$, with a corresponding half-width of $8.0 \%$ for a sample size of 150 patients.

\section{Data analysis}

Summary statistics only are presented for categorical variables (frequency and percentage in category, with $95 \% \mathrm{CI}$ ) and continuous variables (number of subjects, mean, median, standard deviation or standard error, interquartile range, minimum, and maximum). There was no data imputation. 
Table 1. Study population $(N=89)$

\begin{tabular}{|l|l|l|}
\hline \multirow{2}{*}{ Characteristic } & \multicolumn{2}{|c|}{ Patients, $\mathrm{n}(\%)$} \\
\cline { 2 - 3 } & $\begin{array}{l}\text { Very high risk } \\
\text { (n=41) }\end{array}$ & $\begin{array}{l}\text { Extreme risk } \\
\text { (n=48) }\end{array}$ \\
\hline Male/female & $\begin{array}{l}35(85.4 \%) \\
/ 6(14.6 \%)\end{array}$ & $\begin{array}{l}34(70.8 \%) \\
/ 14(29.2 \%)\end{array}$ \\
\hline Age (yrs), mean (range) & $66.1(56-78)$ & $64.3(46-82)$ \\
\hline Weight $(\mathrm{kg})$, & $88.9(57-173)$ & $85.0(49-125)$ \\
mean (range) & {$[\mathrm{n}=32]$} & $\mathrm{n}=38]$ \\
\hline Current smoker & $9(22.0 \%)$ & $10(20.8 \%)$ \\
\hline Diabetes & $6(14.6 \%)$ & $24(50.0 \%)$ \\
\hline STEMI & $12(29.3 \%)$ & $13(27.1 \%)$ \\
\hline Statin-intolerant ${ }^{\mathrm{a}}$ & $1(2.4 \%)$ & $2(4.2 \%)$ \\
\hline Time from diagnosis ${ }^{\mathrm{b}}$ & & \\
$<1$ year & $9(22.0 \%)$ & $13(27.1 \%)$ \\
$\geq 1$ to $<2$ years & $2(4.9 \%)$ & $2(4.2 \%)$ \\
$\geq 2$ to $<3$ years & $1(2.4 \%)$ & $1(2.1 \%)$ \\
$\geq 3$ to $<4$ years & $0(0 \%)$ & $1(2.1 \%)$ \\
$\geq 4$ to $<5$ years & $1(2.4 \%)$ & $1(2.1 \%)$ \\
$\geq 5$ years & $8(19.5 \%)$ & $12(25.0 \%)$ \\
Unknown & $20(48.8 \%)$ & $18(37.5 \%)$ \\
\hline
\end{tabular}

STEMI = ST-elevation myocardial infarction; ${ }^{\text {asymptoms of statin }}$ intolerance; 'time elapsed from diagnosis of hyperlipidemia to study enrolment.

\section{Results}

\section{Study population}

A total of 89 patients, mean age 65.1 (range 46-82) years, were enrolled between December 2016 and March 2017 at four different sites. All were secondary prevention patients, including 30 patients with concomitant diabetes and 25 patients with ST-segment elevation myocardial infarction. There were no FH patients. Forty-eight patients met the criteria for $\mathrm{ER}^{8}$, leaving 41 patients in the VHR group. Patient characteristics are summarized in Table 1, while lifestyle risk factors are listed in Table 2. CV events $(\mathrm{n}=1-7)$ were recorded in 72 (80.9\%) patients, including 33 (80.5\%) VHR and 39 (81.3\%) ER patients during the observation period. During this period, $77(86.5 \%)$ patients were hospitalized, including 35 (85.4\%) VHR and 42 (87.5 \%) ER patients, most commonly because of myocardial infarction $(n=18 ; 20.2 \%)$ or coronary revascularization $(\mathrm{n}=14 ; 15.7 \%)$.

\section{Lipid-lowering therapies}

All patients were receiving statins, mostly as monotherapy (VHR 92.7\%/ER 83.3\%), with very few receiving concomitant LLT (fibrates: VHR 4.9\%/ER 14.6\%

\section{Table 2. Lifestyle factors in study population}

\begin{tabular}{|c|c|c|c|c|}
\hline \multirow[b]{2}{*}{ Parameter } & \multirow[b]{2}{*}{ Category } & \multicolumn{3}{|c|}{ Patients, n (\%) } \\
\hline & & All $(\mathrm{N}=89)$ & $\begin{array}{l}\text { Very high risk } \\
(\mathrm{n}=41)\end{array}$ & $\begin{array}{l}\text { Extreme risk } \\
(\mathrm{n}=48)\end{array}$ \\
\hline Former smoker & $\begin{array}{l}\text { Yes } \\
\text { No } \\
\text { Unknown }\end{array}$ & $\begin{array}{l}28(31.5 \%) \\
26(29.2 \%) \\
35(39.3 \%)\end{array}$ & $\begin{array}{l}12(29.3 \%) \\
11(26.8 \%) \\
18(43.9 \%)\end{array}$ & $\begin{array}{l}16(33.3 \%) \\
15(31.3 \%) \\
17(35.4 \%)\end{array}$ \\
\hline Current smoker & $\begin{array}{l}\text { Yes } \\
\text { No }\end{array}$ & $\begin{array}{l}19(21.3 \%) \\
70(78.7 \%)\end{array}$ & $\begin{array}{l}9(22.0 \%) \\
32(78.0 \%)\end{array}$ & $\begin{array}{l}10(20.8 \%) \\
38(79.2 \%)\end{array}$ \\
\hline Alcohol $\geq 3$ units/day & No & & $41(100.0 \%)$ & $48(100.0 \%)$ \\
\hline Low calorie diet & $\begin{array}{l}\text { Yes } \\
\text { No } \\
\text { Unknown }\end{array}$ & $\begin{array}{l}41(46.1 \%) \\
33(37.1 \%) \\
15(16.9 \%) \\
\end{array}$ & $\begin{array}{l}19(46.3 \%) \\
15(36.6 \%) \\
7(17.1 \%) \\
\end{array}$ & $\begin{array}{l}22(45.8 \%) \\
18(37.5 \%) \\
8(16.7 \%) \\
\end{array}$ \\
\hline Low cholesterol diet & $\begin{array}{l}\text { Yes } \\
\text { No } \\
\text { Unknown }\end{array}$ & & $\begin{array}{l}26(63.4 \%) \\
8(19.5 \%) \\
7(17.1 \%) \\
\end{array}$ & $\begin{array}{l}32(66.7 \%) \\
8(16.7 \%) \\
8(16.7 \%) \\
\end{array}$ \\
\hline Physical activity & $\begin{array}{l}\text { Little/None } \\
\text { Light (1-3 days/week) } \\
\text { Moderate (3-5 days/week) } \\
\text { Very active (6-7 days/week) }\end{array}$ & \begin{tabular}{|l|}
$56(62.9 \%)$ \\
$19(21.3 \%)$ \\
$13(14.6 \%)$ \\
$1(1.1 \%)$
\end{tabular} & $\begin{array}{l}27(65.9 \%) \\
6(14.6 \%) \\
7(17.1 \%) \\
1(2.4 \%)\end{array}$ & $\begin{array}{l}29(60.4 \%) \\
13(27.1 \%) \\
6(12.5 \%) \\
0(0 \%)\end{array}$ \\
\hline
\end{tabular}


Table 3. Summary of mono- and combined lipidlowering therapies (LLT)*

\begin{tabular}{|l|l|l|}
\hline \multirow{2}{*}{ Treatment } & \multicolumn{2}{|c|}{ Patients, $\mathrm{n}(\%)$} \\
\cline { 2 - 3 } & $\begin{array}{l}\text { Very high risk } \\
(\mathrm{n}=41)\end{array}$ & $\begin{array}{l}\text { Extreme risk } \\
(\mathrm{n}=48)\end{array}$ \\
\hline Statin & $38(92.7 \%)$ & $40(83.3 \%)$ \\
Statin + ezetimibe & $1(2.4 \%)$ & $1(2.1 \%)$ \\
Statin + fibrates* & $2(4.9 \%)$ & $7(14.6 \%)$ \\
Total & $41(100 \%)$ & $48(100 \%)$ \\
\hline
\end{tabular}

*Anytime during the observation period. Each subject is included only once (e.g., if they received statin monotherapy for part of the observation period and statin + ezetimibe for another part, they are included under statin + ezetimibe); ${ }^{* *}$ fenofibrate or gemfibrozil.
VHR. The respective levels were $2.4(1.7,3.7) \mathrm{mmol} / \mathrm{L}$ and $2.0(1.5,3.1) \mathrm{mmol} / \mathrm{L}$ for ER. Detailed LDL-C and other lipid data (total and HDL-cholesterol and triglycerides) are shown in Table 6.

During observation, only 17 (41.5\%; 95\% CI 26.32-57.89) VHR patients and 13 (27.1\%; 15.2841.85) ER patients were found to have LDL-C levels within the target ones $(<1.8 \mathrm{mmol} / \mathrm{L}$ and $<1.42$ $\mathrm{mmol} / \mathrm{L}$, respectively) (Fig. 2).

Median LDL-C, total and HDL-cholesterol at the last visit were compared between VHR and ER patients in post-hoc analysis. Only HDL-C showed a (marginally) significant difference between the two

Table 4. Summary of statin use by type and dose*

\begin{tabular}{|l|l|l|l|l|l|}
\hline \multirow{2}{*}{ Type } & Dose $(\mathrm{mg})$ & \multicolumn{2}{|c|}{ Very high risk, n (\%) } & \multicolumn{2}{c|}{ Extreme risk, n (\%) } \\
\cline { 3 - 6 } & & First visit & Last visit & First visit & Last visit \\
\hline \multirow{2}{*}{ Atorvastatin } & 10 & & & $1(2.18 \%)$ & $1(2.18 \%)$ \\
& 20 & $1(2.4 \%)$ & $3(7.3 \%)$ & $3(6.3 \%)$ & $2(4.2 \%)$ \\
& 40 & $5(12.2 \%)$ & $7(17.1 \%)$ & $5(10.4 \%)$ & $6(12.5 \%)$ \\
& 80 & $0(0 \%)$ & $8(19.5 \%)$ & $3(6.3 \%)$ & $7(14.6 \%)$ \\
\hline Fluvastatin & 40 & $1(2.4 \%)$ & $0(0 \%)$ & $0(0 \%)$ & $0(0 \%)$ \\
& 80 & $1(2.4 \%)$ & $0(0 \%)$ & $0(0 \%)$ & $0(0 \%)$ \\
\hline Rosuvastatin & 10 & $1(2.4 \%)$ & $0(0 \%)$ & $1(2.1 \%)$ & $3(6.3 \%)$ \\
& 20 & $3(7.3 \%)$ & $0(0 \%)$ & $4(8.3 \%)$ & $3(6.3 \%)$ \\
& 40 & $2(4.9 \%)$ & $2(4.9 \%)$ & $1(2.1 \%)$ & $0(0 \%)$ \\
\hline Simvastatin & 10 & $1(2.4 \%)$ & $0(0 \%)$ & $0(0 \%)$ & $0(0 \%)$ \\
& 20 & $1(2.4 \%)$ & $0(0 \%)$ & $0(0 \%)$ & $0(0 \%)$ \\
\hline
\end{tabular}

*Data were missing from analysis for approximately $50 \%$ of patients. Patients may have been taking $>1$ statin. Separate analysis showed that $31(75.6 \%)$ very high risk patients and $39(81.3 \%)$ extreme risk patients were taking high-intensity statins (atorvastatin 40-80 mg or rosuvastatin $20-40 \mathrm{mg} /$ day) at some point during the study.

or ezetimibe: VHR 2.4\%/ER 2.1\%) (Table 3); 75.6\% of VHR and $81.3 \%$ of ER patients were taking high-intensity statins (atorvastatin $40-80 \mathrm{mg}$ or rosuvastatin $20-40$ $\mathrm{mg} /$ day) at some point during observation (Table 4).

Thirteen patients (14.6\%: $6 \mathrm{VHR} / 7$ ER) had a switch in LLT during observation and another 13 patients (14.6\%; 5 VHR/8 ER) had modifications in dose and/or frequency, mostly because of insufficient lipidlowering effect, as shown in Table 5. Four patients (all ER) discontinued their LLT for various reasons.

\section{LDL-C and other lipid parameters}

As shown in Figure 1, median (Q1, Q3) LDL-C levels were $2.5(2.0,3.8) \mathrm{mmol} / \mathrm{L}$ at the first and 1.9 $(1.6,2.4) \mathrm{mmol} / \mathrm{L}$ at the last visit of observation for
Table 5. Most common reasons for switching/changing/ discontinuing lipid-lowering therapy (LLT)

\begin{tabular}{|l|l|l|}
\hline \multirow{2}{*}{ Status and reason* } & \multicolumn{2}{|c|}{ Patients, $\mathrm{n}(\%)$} \\
\cline { 2 - 3 } & $\begin{array}{l}\text { Very high risk } \\
(\mathrm{n}=41)\end{array}$ & $\begin{array}{l}\text { Extreme risk } \\
(\mathrm{n}=48)\end{array}$ \\
\hline $\begin{array}{l}\text { Switched to other } \\
\text { LLT (including statins) }\end{array}$ & $6(14.6 \%)$ & $7(14.6 \%)$ \\
$\begin{array}{l}\text { Insufficient lipid } \\
\text {-lowering effect } \\
\text { Modified dose } \\
\text { and/or frequency } \\
\text { Insufficient lipid } \\
\text {-lowering effect } \\
\text { Discontinued }\end{array}$ & $3(50.0 \%)$ & $3(42.9 \%)$ \\
\hline
\end{tabular}

*Reasons expressed as $\%$ of status total; all reasons with $>1$ patient are shown. 
Table 6. Lipid levels (mmol/L) at first and last visit of the observation period

\begin{tabular}{|c|c|c|c|c|}
\hline Parameter & Visit & & Very high risk & Extreme risk \\
\hline \multirow{12}{*}{ LDL-C } & First & $\mathrm{n}$ & 41 & 48 \\
\hline & & Median & 2.510 & 2.350 \\
\hline & & $25^{\text {th }}$ percentile (Q1) & 1.980 & 1.705 \\
\hline & & $75^{\text {th }}$ percentile (Q3) & 3.800 & 3.715 \\
\hline & & Min & 1.3 & 0.7 \\
\hline & & $\operatorname{Max}$ & 6.9 & 5.8 \\
\hline & Last & & 41 & 48 \\
\hline & & Median & 1.900 & 2.030 \\
\hline & & $25^{\text {th }}$ percentile (Q1) & 1.600 & 1.530 \\
\hline & & $75^{\text {th }}$ percentile (Q3) & 2.400 & 3.070 \\
\hline & & $\operatorname{Min}$ & 0.8 & 0.52 \\
\hline & & Max & 4.4 & 4.6 \\
\hline \multirow{16}{*}{ HDL-C } & First & $\mathrm{n}$ & 41 & 48 \\
\hline & & Mean & 1.190 & 1.070 \\
\hline & & Median & 1.140 & 0.995 \\
\hline & & SD & 0.2969 & 0.3120 \\
\hline & & $25^{\text {th }}$ percentile (Q1) & 1.000 & 0.815 \\
\hline & & $75^{\text {th }}$ percentile (Q3) & 1.400 & 1.380 \\
\hline & & Min & 0.70 & 0.50 \\
\hline & & Max & 2.20 & 1.70 \\
\hline & Last & $\mathrm{n}$ & 37 & 45 \\
\hline & & Mean & 1.138 & 1.023 \\
\hline & & Median & 1.100 & 1.000 \\
\hline & & SD & 0.2868 & 0.3134 \\
\hline & & $25^{\text {th }}$ percentile $(\mathrm{Q} 1)$ & 1.000 & 0.800 \\
\hline & & $75^{\text {th }}$ percentile (Q3) & 1.300 & 1.200 \\
\hline & & $\operatorname{Min}$ & 0.60 & 0.50 \\
\hline & & Max & 2.17 & 1.80 \\
\hline \multirow[t]{16}{*}{ Total cholesterol } & First & $\mathrm{n}$ & 40 & 47 \\
\hline & & Mean & 4.840 & 4.638 \\
\hline & & Median & 4.675 & 4.270 \\
\hline & & SD & 1.4478 & 1.5719 \\
\hline & & $25^{\text {th }}$ percentile (Q1) & 3.600 & 3.400 \\
\hline & & $75^{\text {th }}$ percentile (Q3) & 5.850 & 5.500 \\
\hline & & Min & 3.00 & 2.30 \\
\hline & & Max & 9.00 & 8.40 \\
\hline & Last & & 37 & 46 \\
\hline & & Mean & 3.694 & 3.993 \\
\hline & & Median & 3.500 & 3.700 \\
\hline & & & 0.8590 & 1.2131 \\
\hline & & $25^{\text {th }}$ percentile (Q1) & 3.200 & 3.300 \\
\hline & & $75^{\text {th }}$ percentile (Q3) & 3.900 & 4.800 \\
\hline & & Min & 2.10 & 1.70 \\
\hline & & Max & 6.30 & 7.10 \\
\hline \multirow[t]{16}{*}{ Triglycerides } & First & $\mathrm{n}$ & 41 & 47 \\
\hline & & Mean & 1.590 & 1.877 \\
\hline & & Median & 1.600 & 1.600 \\
\hline & & SD & 0.4538 & 0.9145 \\
\hline & & $25^{\text {th }}$ percentile (Q1) & 1.300 & 1.200 \\
\hline & & $75^{\text {th }}$ percentile (Q3) & 1.800 & 2.500 \\
\hline & & Min & 0.84 & 0.60 \\
\hline & & Max & 2.76 & 4.10 \\
\hline & Last & & 38 & 47 \\
\hline & & Mean & 1.384 & 1.708 \\
\hline & & Median & 1.275 & 1.450 \\
\hline & & $\mathrm{SD}$ & 0.6239 & 0.9253 \\
\hline & & $25^{\text {th }}$ percentile (Q1) & 1.100 & 1.000 \\
\hline & & $75^{\text {th }}$ percentile (Q3) & 1.500 & 2.400 \\
\hline & & Min & 0.50 & 0.40 \\
\hline & & Max & 4.40 & 4.30 \\
\hline
\end{tabular}




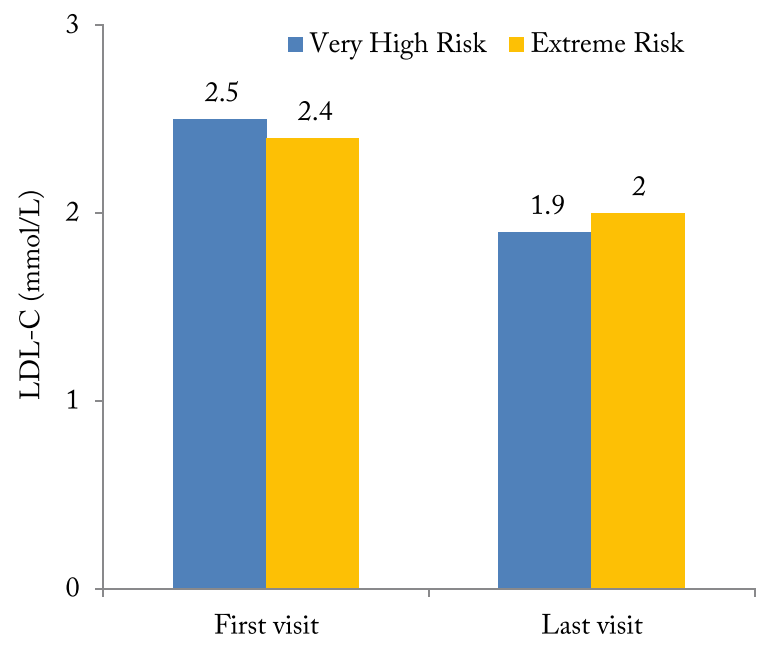

Fig. 1. Median LDL-C levels (mmol/L) at first and last visit of the observation period according to cardiovascular risk category (bars indicate Q1 and Q3).

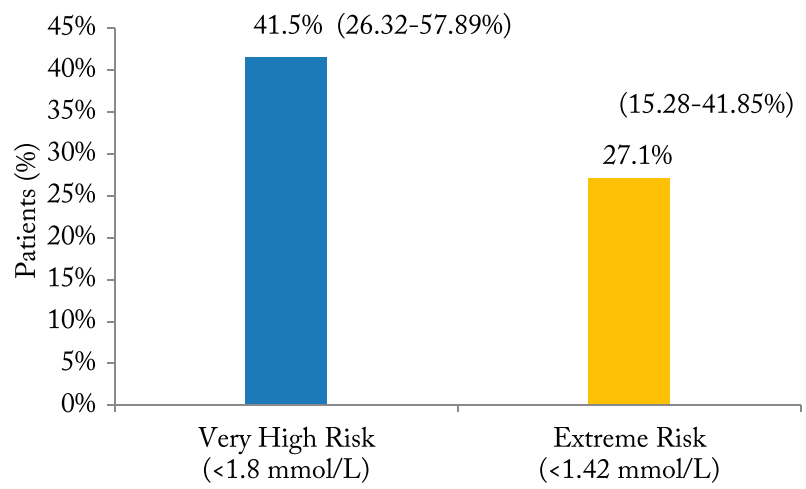

Fig. 2. Achievement of ESC/EAS and AACE-defined $L D L-C$ targets by cardiovascular risk category at any time during the observation period. Results shown as percentage of patients in category with two-sided 95\% confidence intervals in brackets.

subgroups (1.1 in VHR vs. $1.0 \mathrm{mmol} / \mathrm{L}$ in $\mathrm{ER}$; $\mathrm{p}=0.051)$ (Table 7).

\section{Adverse events}

Only three $(<1 \%)$ patients were listed as having symptoms of statin intolerance (statin-associated muscle symptoms (SAMS) or hepatotoxicity).

\section{Discussion}

This observational study explored patterns of management and LDL-C target achievement in VHR
Table 7. Lipid levels at last visit

\begin{tabular}{|c|c|c|c|}
\hline $\begin{array}{l}\text { Parameter } \\
(\mathrm{mmol} / \mathrm{L})\end{array}$ & Statistics & $\begin{array}{l}\text { Very high risk } \\
(\mathrm{N}=41)\end{array}$ & $\begin{array}{l}\text { Extreme risk } \\
(\mathrm{N}=48)\end{array}$ \\
\hline \multirow[t]{9}{*}{ LDL-C } & $\mathrm{N}$ & 41 & 48 \\
\hline & Mean & 2.02 & 2.32 \\
\hline & Median & 1.90 & 2.03 \\
\hline & $\mathrm{SD}$ & 0.68 & 1.01 \\
\hline & Q1 & 1.60 & 1.53 \\
\hline & Q3 & 2.40 & 3.07 \\
\hline & Min & 0.80 & 0.52 \\
\hline & Max & 4.40 & 4.60 \\
\hline & p-value* & 0.30 & \\
\hline \multirow[t]{9}{*}{ HDL-C } & $\mathrm{N}$ & 41 & 48 \\
\hline & Mean & 1.14 & 1.02 \\
\hline & Median & 1.10 & 1.00 \\
\hline & $\mathrm{SD}$ & 0.30 & 0.31 \\
\hline & Q1 & 1.00 & 0.80 \\
\hline & Q3 & 1.30 & 1.20 \\
\hline & Min & 0.60 & 0.50 \\
\hline & Max & 2.17 & 1.80 \\
\hline & p-value* & 0.05 & \\
\hline \multirow[t]{9}{*}{ Triglycerides } & $\mathrm{N}$ & 41 & 48 \\
\hline & Mean & 1.39 & 1.78 \\
\hline & Median & 1.30 & 1.47 \\
\hline & $\mathrm{SD}$ & 0.61 & 1.05 \\
\hline & Q1 & 1.10 & 1.00 \\
\hline & Q3 & 1.50 & 2.40 \\
\hline & Min & 0.50 & 0.40 \\
\hline & Max & 4.40 & 5.30 \\
\hline & $\mathrm{p}$-value* & 0.28 & \\
\hline \multirow[t]{9}{*}{$\begin{array}{l}\text { Total } \\
\text { cholesterol }\end{array}$} & $\mathrm{N}$ & 40 & 47 \\
\hline & Mean & 3.71 & 3.99 \\
\hline & Median & 3.50 & 3.70 \\
\hline & $\mathrm{SD}$ & 0.84 & 1.20 \\
\hline & Q1 & 3.20 & 3.30 \\
\hline & Q3 & 4.10 & 4.80 \\
\hline & Min & 2.10 & 1.70 \\
\hline & Max & 6.30 & 7.10 \\
\hline & $\mathrm{p}$-value ${ }^{*}$ & 0.29 & \\
\hline
\end{tabular}

*Extreme risk vs. very high risk. 
secondary prevention patients with dyslipidemia in Croatia.

Although all patients were receiving statins, mostly at high intensity (75.6\% VHR/81.3\% ER), only a minority were receiving concomitant LLT (7.3\% VHR/16.7\% ER; either ezetimibe or fenofibrate/gemfibrozil). The most common reason for discontinuing or changing LLT during observation was insufficient lipid-lowering effect. Overall, less than half of our patients ( $\mathrm{n}=42,47.2 \%$; 95\% CI 36.5-58.1\%) were found to have LDL-C levels $<1.8 \mathrm{mmol} / \mathrm{L}$ during observation. These proportions were slightly better than those from the main regional study ${ }^{13}$, in which $42.0 \%$ (39.1\%-45.0\%) of VHR patients achieved $<1.8$ $\mathrm{mmol} / \mathrm{L}$. However, our study population differed from the main regional study in that we did not include any FH patients. Approximately half of our patients met the criteria for $\mathrm{ER}^{8}$ and less than $30 \%$ of this subgroup met their very stringent target of $<1.42 \mathrm{mmol} / \mathrm{L}$. It seems likely that such a category will be included in future ESC guidelines.

Underdosing and statin discontinuation/poor adherence to therapy, which can be a consequence of statin intolerance, are recognized as the key contributors to inadequate LDL-C reduction in patients with dyslipidemia and have been linked to poorer clinical outcomes ${ }^{7,14}$. Detailed expert guidelines for diagnosis and management of SAMS are now available and it is hoped that improving awareness of these issues among physicians and patients will have a beneficial effect on statin adherence ${ }^{15}$. Other potential reasons for failure to reach LDL-C targets include reimbursement/financial issues leading to suboptimal treatment, and extremely high baseline LDL-C levels.

The approval in 2015 of the proprotein convertase subtilisin-kexin type 9 inhibitors (PCSK9i) evolocum$\mathrm{ab}$ and alirocumab, which improve LDL-receptor (LDLR) recycling and increase LDLR availability on hepatocyte cell surfaces ${ }^{16}$, has provided new options for treatment of dyslipidemia. Adding PCSK9i can lower LDL-C by additional $60 \%$ in patients who are already receiving maximal statin therapy ${ }^{10,17-20}$, and outcome data have shown that this translates into a significantly reduced risk of $\mathrm{CV}$ events ${ }^{10,21}$. To date, PCSK9i have been well tolerated, with few patients discontinuing treatment due to adverse events ${ }^{10,18,20}$. European guidelines recommend that PCSK9i may be considered for VHR patients with ASCVD, including those with progressive ASCVD or diabetes with target organ damage or a major $\mathrm{CV}$ risk factor or severe $\mathrm{FH}$ without ASCVD but severely elevated LDL-C despite maximal statin/ezetimibe therapy ${ }^{22}$. None of our Croatian patients were receiving these agents.

Limitations of our observational study included small size of some subgroups, such as the statin intolerance group. Changes in LDL-C levels should be interpreted with caution, given the variable duration of LLT, as well as the lack of predefined timepoints for LDL-C measurements. There also were some missing data, e.g., on lifestyle risk factors.

\section{Conclusions}

Our findings indicate that a substantial proportion of VHR and ER secondary prevention patients being treated across Croatia have LDL-C levels exceeding the targets recommended in European and newer AACE/ACE guidelines. Despite this, not all patients are receiving high-intensity statins as recommended. Identification of ER patients and their lipid patterns may help optimize the usage of high-intensity statin treatment, alone or in combination with newer treatments, for better control of elevated LDL-C.

Data availability. The datasets used and/or analyzed during the study are available from the corresponding author on reasonable request. Qualified researchers may request data from Amgen clinical studies. Complete details are available at: http://www.amgen.com/ datasharing.

\section{Acknowledgments}

The study was supported by Amgen. Joshua David of Quartesian, Bangalore, provided statistical analysis. Julia Balfour of Northstar Medical Writing and Editing, Dundee, UK, provided medical writing support (with financial support from Amgen).

The authors wish to thank the following participating centers, their patients and staff: Sestre milosrdnice University Hospital Centre, Department of Cardiovascular Diseases, Zagreb; Zagreb University Hospital Centre, Department of Cardiovascular Diseases, $\mathrm{Za}^{-}$ greb; Osijek University Hospital Centre, Department of Cardiology, Osijek; and Rijeka University Hospital Centre, Department of Cardiology, Rijeka. 


\section{References}

1. World Health Organization. Cardiovascular diseases. Fact sheet. Updated May 2017. http://www.who.int/mediacentre/ factsheets/fs317/en/In. 2017.

2. Kannel WB. The Framingham Study: an epidemiological investigation of cardiovascular disease, Section 30. Some characteristics related to the incidence of cardiovascular disease and death: The Framingham Study. 18-year follow-up.Washington, D.C., Department of Health, Education, and Welfare, Publication No. (NIH) 74-599, 1974.

3. Kannel WB. Range of serum cholesterol values in the population developing coronary artery disease. Am J Cardiol. 1995;76: 69C-77C. http://dx.doi.org/10.1016/s0002-9149(99)80474-3.

4. Kannel WB, Castelli WP, Gordon T. Cholesterol in the prediction of atherosclerotic disease: new perspectives based on the Framingham Study. Ann Intern Med. 1979;90:85-91. http:// dx.doi.org/10.7326/0003-4819-90-1-85.

5. Cholesterol Treatment Trialists Collaboration. Efficacy and safety of more intensive lowering of LDL cholesterol: a metaanalysis of data from 170,000 participants in 26 randomised trials. Lancet. 2010;376:1670-81. http://dx.doi.org/ 10.1016/ S0140-6736(10)61350-5

6. Reiner Z, Catapano AL, De Backer G, et al. ESC/EAS Guidelines for the Management of Dyslipidaemias: The Task Force for the Management of Dyslipidaemias of the European Society of Cardiology (ESC) and the European Atherosclerosis Society (EAS). Eur Heart J. 2011;32:1769-818. http://dx.doi. org/ 10.1093/eurheartj/ehr158.

7. Catapano AL, Graham I, De Backer G, et al. 2016 ESC/EAS Guidelines for the Management of Dyslipidaemias: The Task Force for the Management of Dyslipidaemias of the European Society of Cardiology (ESC) and European Atherosclerosis Society (EAS). Developed with the special contribution of the European Assocciation for Cardiovascular Prevention \& Rehabilitation (EACPR). Atherosclerosis. 2016;253:281-344. http://dx.doi.org/ 10.1016/j.atherosclerosis.2016.08.018.

8. Jellinger PS, Handelsman Y, Rosenblit PD, et al. American Association of Clinical Endocrinologists and American College of Endocrinology Guidelines for Management of Dyslipidemia and Prevention of Cardiovascular Disease. Endocr Pract. 2017;23:1-87. https://doi.org/10.4158/EP171764.APPGL.

9. Cannon CP, Blazing MA, Giugliano RP, et al. Ezetimibe added to statin therapy after acute coronary syndromes. N Engl J Med. 2015;372:2387-97. http://dx.doi.org/10.1056/NEJMoa1410489.

10. Sabatine MS, Giugliano RP, Keech AC, et al. Evolocumab and clinical outcomes in patients with cardiovascular disease. $\mathrm{N}$ Engl J Med. 2017;376:1713-22. http://dx.doi.org/10.1056/ NEJMoa1615664.

11. Marrett E, Zhao C, Zhang NJ, et al. Limitations of real-world treatment with atorvastatin monotherapy for lowering LDL-C in high-risk cardiovascular patients in the US. Vasc Health Risk Manag. 2014;10:237-46. http://dx.doi.org/ 10.2147/ VHRM.S54886.

12. Steinberg BA, Bhatt DL, Mehta S, et al. Nine-year trends in achievement of risk factor goals in the US and European outpatients with cardiovascular disease. Am Heart J.2008;156:71927. https://doi.org/10.1016/j.ahj.2008.05.020.

13. Petrov I, Dumitrescu A, Snejdrlova M, et al. Clinical management of high and very high risk patients with hyperlipidaemia in Central and Eastern Europe: an observational study. Adv Ther. 2019;36:608-20. https://doi.org/10.1007/s12325-0190879-1.

14. Banach M, Stulc T, Dent R, Toth PP. Statin non-adherence and residual cardiovascular risk: there is need for substantial improvement. Int J Cardiol. 2016;225:184-96. https://doi. org/10.1016/j.ijcard.2016.09.075.

15. Stroes ES, Thompson PD, Corsini A, et al. Statin-associated muscle symptoms: impact on statin therapy - European Atherosclerosis Society Consensus Panel Statement on Assessment, Aetiology and Management. Eur Heart J. 2015;36:101222. https://doi.org/10.1093/eurheartj/ehv043.

16. Seidah NG, Awan Z, Chrétien M, Mbikay M. PCSK9. A key modulator of cardiovascular health. Circ Res. 2014;114:102236. http://doi.org/10.1161/CIRCRESAHA.114.301621.

17. Lipinski MJ, Benedetto U, Escarcega RO, et al. The impact of proprotein convertase subtilisin-kexin type 9 serine protease inhibitors on lipid levels and outcomes in patients with primary hypercholesterolaemia: a network meta-analysis. Eur Heart J. 2016;37:536-45. http://doi.org/10.1093/eurheartj/ ehv563.

18. Navarese EP, Kolodziejczak M, Schulze V, et al. Effects of proprotein convertase subtilisin/kexin type 9 antibodies in adults with hypercholesterolemia: a systematic review and meta-analysis. Ann Intern Med.2015;163:40-51. http://doi.org/10.7326/ M14-2957.

19. Sabatine MS, Giugliano RP, Pedersen TR. Evolocumab in patients with cardiovascular disease. N Engl J Med. 2017;377: 787-8. http://doi.org/10.1056/NEJMc1708587.

20. Sabatine MS, Giugliano RP, Wiviott SD, et al. Efficacy and safety of evolocumab in reducing lipids and cardiovascular events. N Engl J Med. 2015;372:1500-9. http://doi.org/ 10.1056/NEJMoa1500858.

21. Robinson JG, Farnier M, Krempf M, et al. Efficacy and safety of alirocumab in reducing lipids and cardiovascular events. N Engl J Med. 2015;372:1489-99. http://doi.org/10.1056/ NEJMoa1501031.

22. Landmesser U, Chapman MJ, Stock JK, et al. 2017 Update of ESC/EAS Task Force on practical clinical guidance for proprotein convertase subtilisin/kexin type 9 inhibition in patients with atherosclerotic cardiovascular disease or in familial hypercholesterolaemia. Eur Heart J. 2018;39(14):1131-43. https:// doi.org/10.1093/eurheartj/ehx549 


\section{Sažetak \\ LIJEČENJE HIPERLIPIDEMIJE U BOLESNIKA S VRLO VISOKIM I EKSTREMNO VISOKIM RIZIKOM U HRVATSKOJ}

\section{H. Pintaric, M. Knezović Florijan, I. Bridges, R. Steiner, L. Zaputović i D. Miličić}

Ova opservacijska studija analizirala je trenutno liječenje povišenih vrijednosti LDL kolesterola (LDL-C) u bolesnika koji su liječeni zbog hiperlipidemije u okviru sekundarne prevencije (prema europskim smjernicama svi su pripadali u skupinu bolesnika s vrlo visokim rizikom) i kontrolirali su se kod specijalista diljem Hrvatske. Podaci su prikupljeni retrospektivno tijekom 12 mjeseci iz bolesničke medicinske dokumentacije. Skupina bolesnika za koju je procijenjeno da je u ekstremno visokom riziku (ER; AACE kriteriji; $n=48$ ) uspoređivana je sa skupinom s visokim rizikom (VHR skupina; $n=41$ ). Svi bolesnici su primali statine $(75,6 \%$ bolesnika s $\mathrm{VHR} / 81,3 \%$ bolesnika s ER liječeno je visokim dozama statina), dok je manjina bolesnika istodobno primala i drugu terapiju za snižavanje lipida (7,3\% VHR/ER 16,7\%). Kod posljednjeg posjeta medijan vrijednosti LDL-C (Q1, Q3) u skupini VHR iznosio je 1,9 $(1,6,2,4) \mathrm{mmol} / \mathrm{L}$, dok je u skupini ER iznosio 2,1 $(1,5,3,1)$ mmol/L. Samo 41,5\% (95\% CI 26,3-57,9) bolesnika s VHR i 27,1\% (15,3-41,9) bolesnika s ER imali su razine LDL-C unutar ciljne razine $(<1,8 \mathrm{mmol} / \mathrm{L} \mathrm{i}<1,42 \mathrm{mmol} \mathrm{L})$. Rezultati studije pokazuju kako unatoč mjerama sekundarne prevencije velik broj visokorizičnih bolesnika i bolesnika s ekstremnim rizikom ima razine LDL-C koje premašuju ciljne vrijednosti preporučene u europskim i novijim AACE smjernicama, ali usprkos navedenom mnogi bolesnici nisu liječeni statinima $u$ visokoj dozi. Identifikacija bolesnika s ekstremnim rizikom i njihovih lipidnih obrazaca mogla bi povećati upotrebu visokih doza statina, samostalno ili u kombinaciji s novijim lijekovima, radi bolje kontrole povišenog LDL-C

Ključne riječi: Hiperlipidemija; Sekundarna prevencija; Bolesnici s visokim rizikom; Bolesnici s vrlo visokim rizikom; Bolesnici sekstremno visokim rizikom 